\title{
Hydrophilic Gold Supracrystals Differing by the Nanoparticle Crystalline Structure
}

\author{
Stefanos Mourdikoudis ${ }^{a, b}$, Arzu Çolak $^{a, b}, \operatorname{Imad}_{\text {Arfaoui }^{a}, \mathbf{b}}$, Marie-Paule Pileni ${ }^{\mathrm{a}, \mathrm{b}, \mathrm{c}, \text { * }}$ \\ ${ }^{a}$ Sorbonne Universités, UPMC Univ Paris 06, UMR 8233, MONARIS, F-75005, Paris, France \\ ${ }^{b}$ CNRS, UMR 8233, MONARIS, F-75005 Paris, France \\ ${ }^{c}$ CEA/IRAMIS, CEA Saclay, 91191, Gif-sur-Yvette, France
}

\begin{abstract}
Very few studies concern water-soluble nanocrystals self-assembled in crystalline 3D superlattices called supracrystals. Furthermore, the control of the crystalline structure of nanocrystals known as nanocrystallinity has not been yet achieved with water-soluble nanocrystals. Here we produce, selectively, 5-nm Au single domain (SD) and polycrystalline (POLY) water-soluble nanocrystals. These nanocrystals self-assembled in face-centered-cubic (fcc) supracrystals. The supracrystal stiffness evolves with the nanocrystallinity, the nanocrystal surface charge as well as the steric effect of the coating agent. The optical properties of SD and POLY nanoparticles and those of the related supracrystals are also presented. In addition, a nanocrystallinity segregation event was observed upon drying-assisted self-assembly of aqueous stoichiometric mixtures of SD and POLY NCs, as in the case of their hydrophobic counterparts.
\end{abstract}

\section{Introduction}

A significant progress was observed during the last two decades in the investigation of ways for the self-assembly of hydrophobic inorganic nanoparticles (e.g. metals, metal oxides, metal chalcogenides) in two or three-dimensional (2D or 3D) crystals ${ }^{1,2}$ as such structures show a tremendous interest due to their potentially newly arising inherent collective properties. ${ }^{3-6}$ When nanocrystals (NCs) are arranged in 2D superlattices collective optical and/or magnetic properties thanks to dipolar interactions may be observed. ${ }^{3}$ In the case of the formation of supracrystals (SCs), that is, 3D arrays of self-organized nanoparticles (NPs), new mechanical, vibrational and magnetic properties can be acquired. ${ }^{4,5,6}$ These supracrystals demonstrate well-defined crystalline structures, being composed of $\mathrm{NCs}$, in a similar way to the fact that bulk materials are consisted of atoms. ${ }^{7-9}$ The spherical NPs having low size distribution packed in compact hexagonal networks are responsible for the formation of such (SCs), which may possess several types of arrangements (i.e. fcc, hcp or bcc)..$^{7,10}$ The control of both interparticle distance and surface chemistry is a key factor for the ability to tune their assembly properties. Very recently, several groups studied the influence of the crystalline structure of NCs (single domain and polycrystals) called nanocrystallinity. Still some contrevercies exist. However, a marked change was demonstrated in some SCs physical properties (magnetic, ${ }^{11}$ mechanical, ${ }^{12}$ surface structure, ${ }^{13}$ ) with nanocrystallinity. Such novel 'tailored' materi draw attention from the academic point of view, ${ }^{14,15}$ but also with regard to applications in domains as optoelectronics, ${ }^{16}$ bio-sensing, ${ }^{17}$ magnetic storage, ${ }^{18}$ catalysis $^{19}$ and chemical amplifiers. ${ }^{20}$ Such specific properties concerning the aforementioned supracrystals involve hybrophobic $3 \mathrm{D}$ superlattices.

Syntheses of water-soluble NCs with low size distribution are somewhat less developed compared to the ones in organic medium. ${ }^{20-}$ ${ }^{26}$ Recently, we revised ${ }^{27}$ a synthetic method developed elsewhere ${ }^{28}$ based on ligand exchange processes. NCs with low size distribution differing by their coating agents and their charges were produced. By using a single set of NCs, it has been demonstrated that both film and shaped SCs are grown. In addition, water soluble Au nanocrystals were shown to be biocompatible nanomaterial with potential applications in drug delivery, ${ }^{24}$ diagnostic and imaging. ${ }^{25,}{ }^{26}$ More versatile functions could be expected if these biocompatible NPs assemble into supracrystals.

During the last two decades it has been demonstrated that supracrystals production needs (i) a control of the NC size distribution leading to the development of a large variety of chemical pathways. ${ }^{29-}$ 31 and (ii) a slow solvent evaporation process. ${ }^{32} \mathrm{~A}$ low NC size distribution to produce well-defined films and shaped supracrystals is therefore necessary, but the control of nanocrystallinity is also crucial. Very recently we demonstrated that such control is obtained by producing separately populations of single domain (SD) and polycrystalline (POLY) NPs, taking Au system as an example. This was obtained by keeping a solution of hydrophobic Au nanocrystals under solvent vapor saturation during more than 7 days. The SD nanocrystals formed both precipitate and interfacial film. ${ }^{9}$ Current syntheses producing a given kind of nanocrystallinity (either SD or (POLY)) are quite limited. ${ }^{9}, 32,33,34,36$ 
Herein we present a procedure for the preparation of water-soluble $\mathrm{Au}$ NCs with either single-domain or polycrystalline structure. The initial particles used were hydrophobic 5-nm Au nanocrystals which were separated in two populations using a nanocrystallinity segregation process. ${ }^{36} \mathrm{We}$ then developed a new ligand exchange protocol to render them hydrophilic. The final water-soluble NCs retain their initial size and size distribution with specific nanocrystallinity either SD or POLY NCs. The resulting 5-nm Au NCs are self-assembled in 3D-films by using positively-charged ligand (ammonium chloride). The morphology, optical properties and interparticle distance of the films are characterized by several techniques, and the mechanical properties between the SD and POLY NC-based films are compared. Finally, in the case of supracrystal films which are produced from stoichiometric colloidal mixtures of $\mathrm{SD}$ and POLY NCs, a nanocrystallinity segregation process is highlighted.

\section{Results and Discussion \\ II.1. Production of water soluble NCs}

The 5-nm Au NCs coated with dodecanethiol (DDT) molecules were produced by a revisited method from the Stucky group (see experimental part ${ }^{37}$ and a mixture of SD and POLY AuDDT NCs were obtained (Figure 1a). The average diameter and size distribution were $5 \mathrm{~nm}$ and $0.2 \mathrm{~nm}$ respectively (Figure 1b). As described elsewhere, ${ }^{9}$ in order to induce nanocrystallinity selection a colloidal solution of AuDDT NCs was maintained, during a week, in a closed flask under solvent vapor saturation. It has been demonstrated ${ }^{36}$ that the obtained black precipitate was composed of single domain AuDDT NCs (SDAuDDT) whereas the supernatant contained its polycrystalline counterpart (POLYAUDDT). This claim was confirmed by the bright (Figures 1c and 1e) and dark (Figures 1d, 1f) field TEM images of POLYAuDDT and SDAuDDT NCs respectively. The dark field image of POLYAuDDT NCs showed heterogenous contrast (Figure 1d) whereas that of SDAuDDT NCs is highly homogeneous with either a totally bright or dark color, or a 'hollow'-looking contrast (Figure 1f).

DDT used as a coating agent for the initially prepared NCs was attempted to be replaced by N,N,N-trimethyl(11-mercaptoundecyl) ammonium chloride (TMA) molecules by first trying to use a ligand exchange process developed elsewhere. ${ }^{27}$ However, contrary to what was expected, the result was not successful; also conventional protocols including room-temperature stirring overnight, ${ }^{38,39}$ led to irreversible aggregation of the NCs. To tackle this problem, we combined magnetic stirring $(700 \mathrm{rpm})$ with heating the toluenedispersed NPs at a temperature of $80{ }^{\circ} \mathrm{C}$ for $3 \mathrm{~h}$. At the end of the ligand exchange process, SD and POLY 5-nm Au NCs coated with TMA and denoted SDAuTMA and POLYAuTMA NCs, respectively, were produced separately. The average diameter of SDAuTMA and POLYAutMa NCs were 5.4 and $5.2 \mathrm{~nm}$ respectively with $0.2-\mathrm{nm}$ as standard deviation of size. These Autma NCs dispersed in aqueous solution were characterized by a deep red color. These colloids were stable for several months. AuTMA NCs, due to the nature of the TMA molecule, were positively charged, as expected. ${ }^{27}$

The TEM images presented in Figures $2 \mathrm{a}$ and $2 \mathrm{c}$ show that after transfer into aqueous solution, both SDAuTMA and POLYAuTMA NCs remained unchanged in size (average diameters 5.4-nm and 5.2-nm respectively) and size distribution (around 5\%). The difference in contrast between SD (Figure 2b) and POLY (Figure 2d) NCs was kept unmodified as observed for their counterparts coated with DDT (Figures 1d and 1f) with homogeneous and heterogeneous contrast patterns, correspondingly.

The colloidal solution of SDAuTMA and POLYAutma NCs dispersed in aqueous solution is characterized by a well-known localized surface plasmon resonance (LSPR) centered at $520 \mathrm{~nm}$ (Figure 3). The
POLYAuTMA NCs LSPR spectrum has a larger linewidth, compared to that produced with SDAuTMA NCs. Note that the position of the peak remained unchanged. These data agreed with those previously obtained with 5-nm AuDDT NCs. ${ }^{40}$ As in the case 5-nm Au DDT NCs, ${ }^{40}$ the LSPR band of POLYAuTMA NCs possesses a lower intensity and a larger linewidth, compared to their monocrystalline counterparts. Taking into account that both samples consisted of NCs with the same size, the slight differences in their LSPR spectra were due to twin defects as already proposed elsewhere. ${ }^{35,41}$

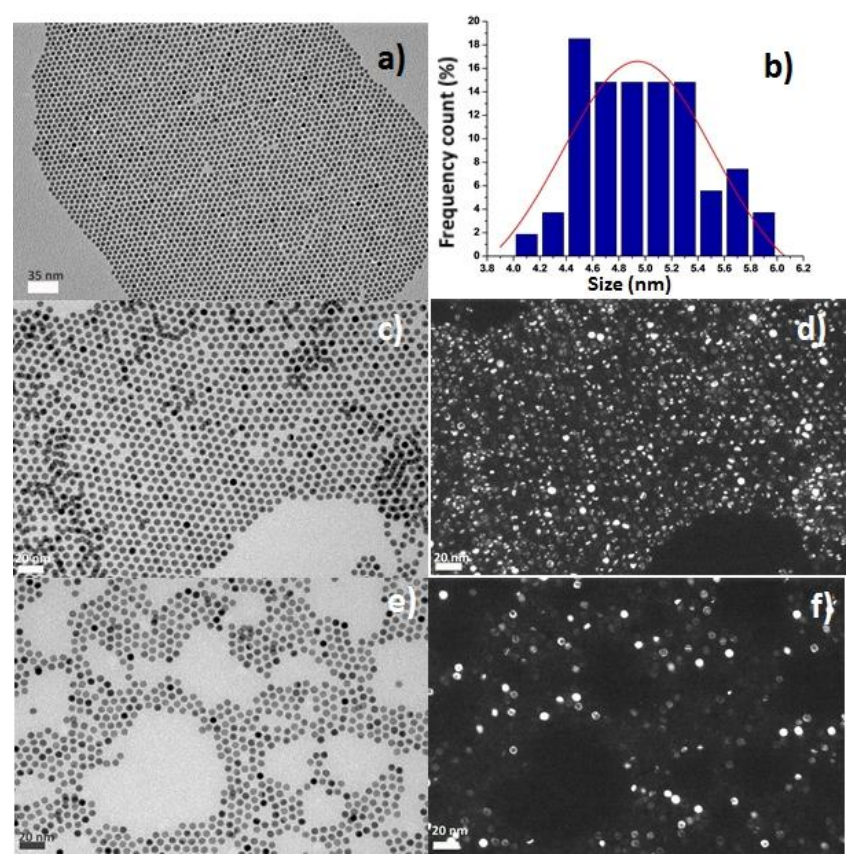

Figure 1. a) Bright-field TEM image of 5-nm Au NCs coated with DDT $\left(\mathrm{Au}_{\mathrm{DDT}}\right)$ and dispersed in toluene, $\left.\mathrm{b}\right)$ the corresponding size distribution histogram, (c and d) POLYAu ${ }_{\text {DDT }} \mathrm{NCs}$, TEM bright- $\mathrm{c}$ ) and dark-field d) images. $(\mathrm{e}, \mathrm{f}){ }_{\mathrm{SD}} \mathrm{Au}_{\mathrm{DDT}} \mathrm{NCs}$, Scale bars: a) $35 \mathrm{~nm}$ and c)-f) $20 \mathrm{~nm}$.

\section{II.2. Facet-facet interactions in the thin films of NCs differing by their nanocrystallinity}

A drop of concentrated $(6 \mathrm{mM})$ aqueous colloidal solutions of each of the two samples (sDAutma and polyAutma NCs) was deposited onto a TEM grid. At the end of the water evaporation process (several hours) self-assembled NC structures with a thickness of a few NC layers (Figures $2 \mathrm{~g}$ and $2 \mathrm{i}$ ) were produced. The high-angle electrondiffraction (HAED) patterns of both assemblies (sDAutma or POLYAutma NCs) showed several rings assigned to planes of atomic lattices with fcc crystal structures (Figures $2 \mathrm{~h}$ and $2 \mathrm{j}$ ). Hence, whatever the nanocrystallinity, the sDAuTMA and POLYAuTMA NCs were assembled in fcc crystalline structure. Actually, with SDAuTMA NCs films, small arcs were clearly spotted for some of the diffraction rings (Figure 2h). In contrast, with POLYAuTMA NCs films, Figure $2 \mathrm{j}$ showed no arcs. Presence and absence of arcs respectively were confirmed from the first diffraction ring profile (counting from the center of the HAED images outwards) of SDAuTMA NCs- and POLYAutMA NCs films (Figure 4) as observed previously with 5-nm SDAuDDT and POLYAutma NCs films. ${ }^{36}$ Similar behavior has been observed before, attributing presence of arcs to facet-facet interactions with an average coherent alignment of the atomic lattice planes of NCs to one another within the superlattice. ${ }^{40-44}$ Such facetfacet interactions are explained by the fact that SD NCs are characterized by larger facets than POLY NCs as observed in Figures $5 \mathrm{a}$ and $5 \mathrm{~b}$. By analogy, we claimed that orientational ordering of the atomic lattices planes of SDAuTMA NCs is indicative of coherent 
alignment with those of adjacent NCs through their faceted interactions. The latter is verified by their partial preference to form faceted shapes as depicted by corresponding HRTEM images (Figure 5) promoting orientational alignment via face-to-face interactions. On the other hand, the quasi-spherical shape of poLYAuTMA NCs (Figure $5 \mathrm{~b}$ and $5 \mathrm{~d}$ ) was related to their tendency to be self-assembled with long-range translational order, while the SDAuTMA NCs present both translational and orientational ordering upon their assembly. In fact, a closer look by HRTEM at the POLYAuTMA NCs illustrated that some of them are multiply-twinned with tetrahedral sub-units and shapes such as decahedron and icosahedron (Figure S1 in Supporting Information). These data are rather surprising if we take into account that the coating agents (TMA) have ammonium as terminal group, positively charged. We would expect strong electrostatic repulsion between NCs. From that it is concluded that the facet-facet interactions combined with the van der Waals ones remain strong enough to compete with the electrostatic repulsive force and consequently favoring the observation of orientational ordering.
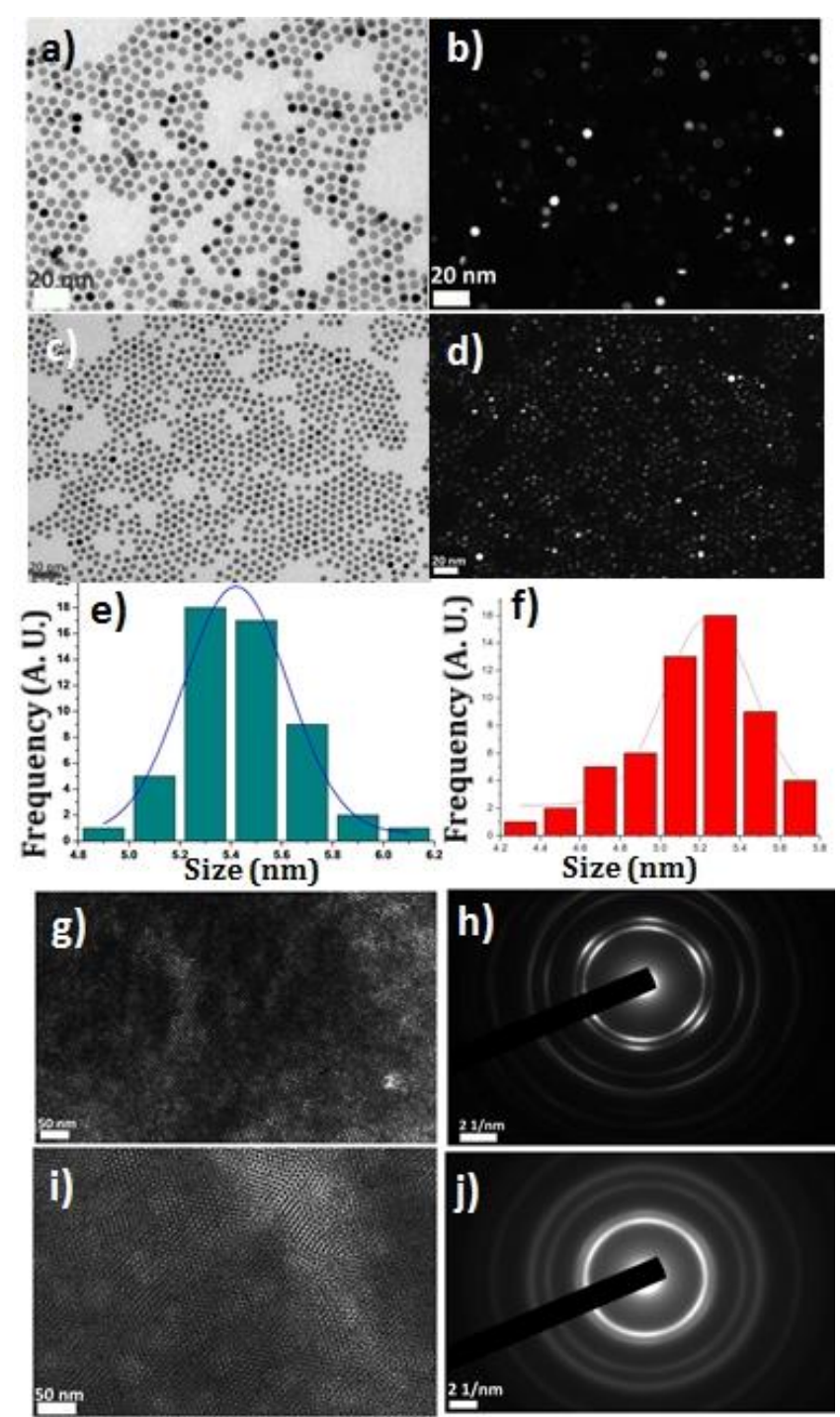

Figure 2. a) and b): Bright- and dark-field TEM images of water-soluble 5$\mathrm{nm}_{\mathrm{SD}} \mathrm{Au}_{\mathrm{TMA}}$ NCs. c) and d) Bright- and dark field TEM images of ${ }_{\mathrm{POLY}} \mathrm{Au}_{\mathrm{TMA}}$ NCs. e) and f): Size distribution curves of ${ }_{S D} \mathrm{Au}_{\mathrm{TMA}}$ and ${ }_{\mathrm{POLY}} \mathrm{Au}_{\mathrm{TMA}} \mathrm{NCs}$ respectively. g) and $\mathrm{h}$ ) Thin film (few layers) of ${ }_{\mathrm{SD}} \mathrm{Au}_{\mathrm{TMA}} \mathrm{g}$ ) and ${ }_{\mathrm{POLY}} \mathrm{Au}_{\mathrm{TMA}} \mathrm{i}$ ) NCs and their $h$ ), j), corresponding high-angle electron-diffraction (HAED) pattern respectively.

\section{3. Growth of thick supracrystals}

On increasing the NCs concentration $([\mathrm{Au}]=10 \mathrm{mM}), 100 \mu \mathrm{l}$ of each dispersion (either SDAuTMA or POLYAuTMA NCs) was deposited in a glass beaker with a hydrophilic silicon wafer at its bottom. The beaker was introduced in a pre-heated oven at $60^{\circ} \mathrm{C}$, in high humidity environment. Water was slowly evaporated (within $48 \mathrm{~h}$ ) and a film with bright gold color was formed onto the substrate. Figures $6 \mathrm{a}$ and $6 \mathrm{~b}$ show that the SDAuTMA NCs generate ordered film supracrystals upon their assembly.

Representative HRSEM images (Figures 6c, S2 and S3) as well as typical fast-Fourrier-transformed patterns (Figure 6d) illustrate that the SDAutma NCs formed a hexagonal arrangement. The POLYAuTMA NCs show similar features, although a tendency to form 'steps' during the formation of supracrystals was somewhat more evident in that case (Figures 6e, 6f, S4 and S5). Actually a closer look by HRSEM at the SDAuTMA NCs films reveals that some step-like growth occurs also in that case (Figure S3a). Overall, such images depict NC arrays with distinguishable step edges and homogeneous orientation. Smallangle X-ray diffraction (SAXRD) patterns (Figure 7) confirmed that both sDAutma NCs and POLYAutma NCs produce (fcc) crystalline films.

The average edge-to-edge interparticle distance, $\delta$, calculated from the $\mathrm{d}_{(111)}$ spacing at the SAXRD curves is 2.2-nm and 2.4-nm for SDAuTMA and POLYAuTMA NCs, respectively. These values are slightly higher than the molecular length of TMA $(1.9 \mathrm{~nm})$. As already mentioned such interdigitation is rather surprising if we take into account that Autma NCs are positively charged. The influence of the facet-facet interactions, observed above with thin films, is confirmed if we consider that interparticle distance between SDAuTMA NCs is slighltly shorter than that obtained for POLYAuTMA NCs.

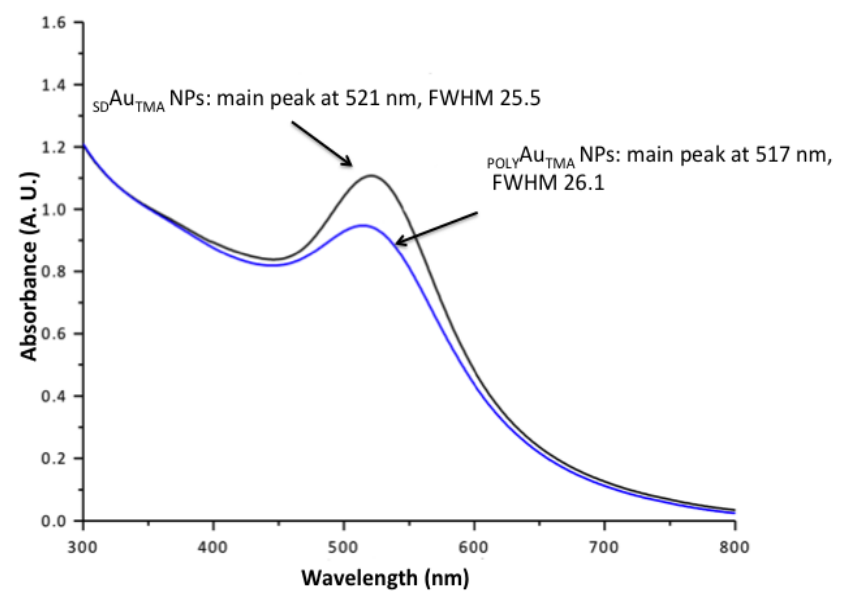

Figure 3. UV-Vis spectra of $5 \mathrm{~nm}_{\mathrm{SD}} \mathrm{Au}_{\mathrm{TMA}}$ and POLYAu $\mathrm{TMA}_{\mathrm{TMA}} \mathrm{NCs}$.

II. 4 Segregation process induced by mixing polyAutMa and SDAutma NCs

Two colloidal solutions (equiatomic 50/50) of POLYAuTMA and SDAuTMA NCs respectively were mixed and $100 \mu$ of such resulting stoichiometric solution was deposited in a beaker as described above. By slow evaporation process, a thick film was deposited onto a silicon substrate. The SEM images (Figures $8 \mathrm{a}$ and $8 \mathrm{~b}$ ) and the corresponding HRSEM images (Figure S6) show various zones with either large or small surface area and cracks.

Instead of depositing a large amount of the colloidal solution, a drop $(10 \mu \mathrm{l})$ was dispersed onto a TEM grid. The drying process was obtained by keeping overnight the sample in air, at room temperature, and a film with a thickness of a few NCs layers was produced (Figures $8 \mathrm{c}$ and $8 \mathrm{e}$ ). The respective high-angle electron-diffraction (HAED) 
pattern of the two different regions corresponding to Figures $8 \mathrm{c}$ and 8e exhibit several rings corresponding to electron diffraction by specific plane of atomic lattices with fcc structure. The HAED pattern corresponding to Figure $8 \mathrm{c}$ showed small arcs (Figure 8d), as observed for the film produced with SDAuTMA NCs (Figure $2 \mathrm{~h}$ ), for most of the diffraction rings whereas no arcs were observed in the HAED pattern (Figure 8f) corresponding to the image of Figure 8e. As already mentioned, the presence of arcs provide evidence for an average coherent alignment of the atomic lattice planes of NCs to one another within the superlattice. Such orientational ordering of the atomic lattices of SDAuTMA NCs is indicative of their faceted morphology and is presumably favored by large facets in singledomain NCs, as noted above.

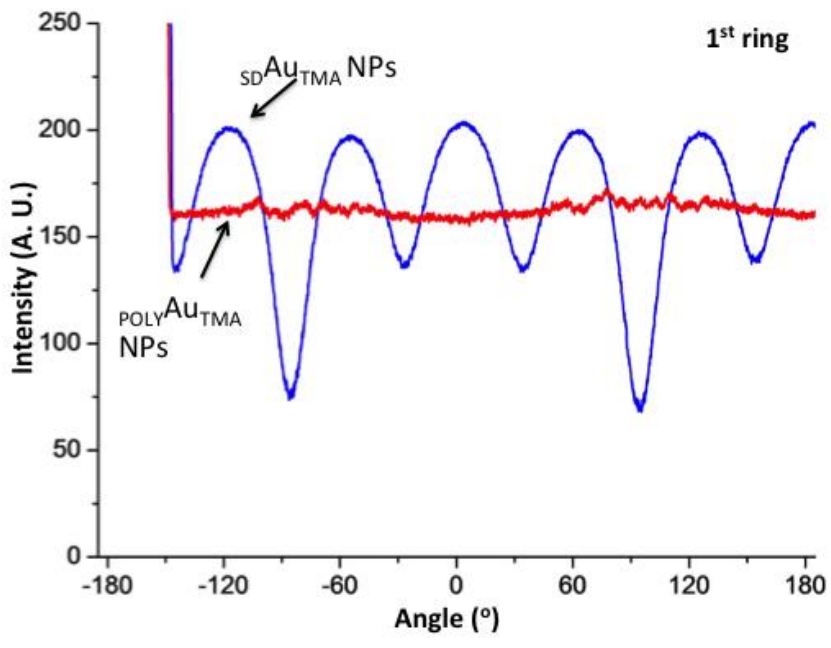

Figure 4. Profiles for first diffraction rings of electron diffraction patterns of 4- to 5- layer-thick superlattices of ${ }_{\mathrm{SD}} \mathrm{Au}_{\mathrm{TMA}}$ (blue curve) and ${ }_{\mathrm{POLY}} \mathrm{Au}_{\mathrm{TMA}} \mathrm{NCs}$ (red curve)

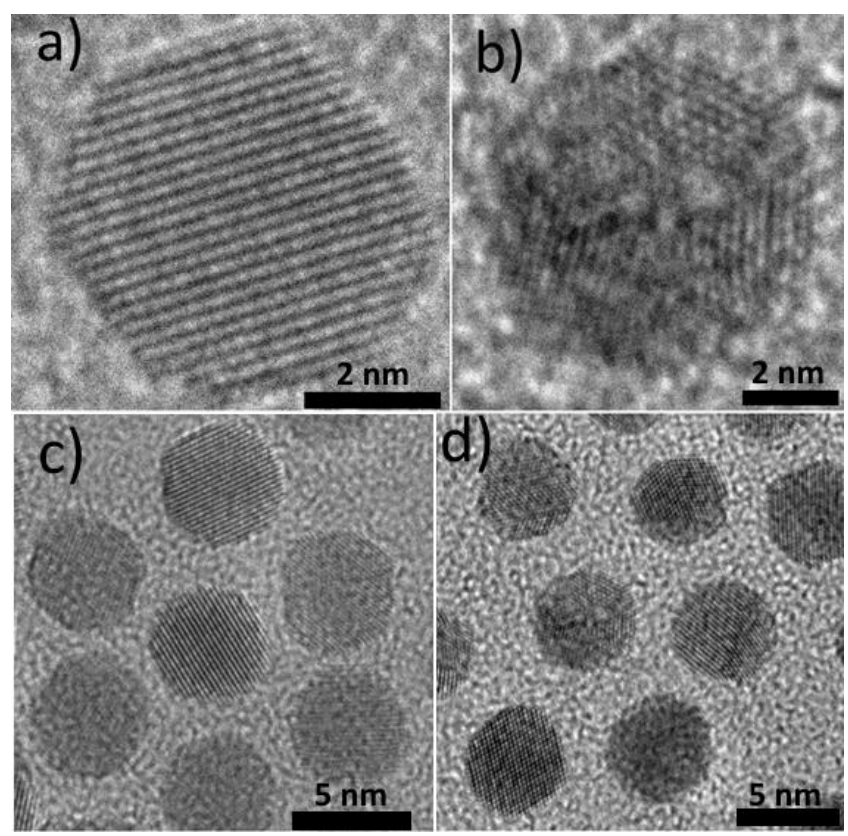

Figure 5. High-resolution TEM images of ${ }_{\mathrm{SD}} \mathrm{Au}_{\mathrm{TMA}}(\mathrm{a}, \mathrm{c})$ and ${ }_{\mathrm{POLY}} \mathrm{Au}_{\mathrm{TMA}}(\mathrm{b}, \mathrm{d})$ $\mathrm{NCs}$ either isolated $(\mathrm{a}, \mathrm{b})$ or self assembled in 2D superlattices $(\mathrm{c}, \mathrm{d})$.

On the other hand, the absence of arcs (Figure 8f) implies selfassembly into arrays with only long range translational (not orientational) ordering. These data clearly demonstrate that, during the deposition process (slow evaporation), two distinct types of regions exist: one is composed by mainly SDAuTMA NCs whereas the second one contains a rich population of POLYAuTMA NCs. Recently, ${ }^{40}$ we observed similar data with hydrophobic 5-nm Au nanocrystals coated with tetradecanethiol (TDT), where we noticed formation of zones containing mainly SDAuTDT NCs while other areas were composed of POLYAuTDT NCs.
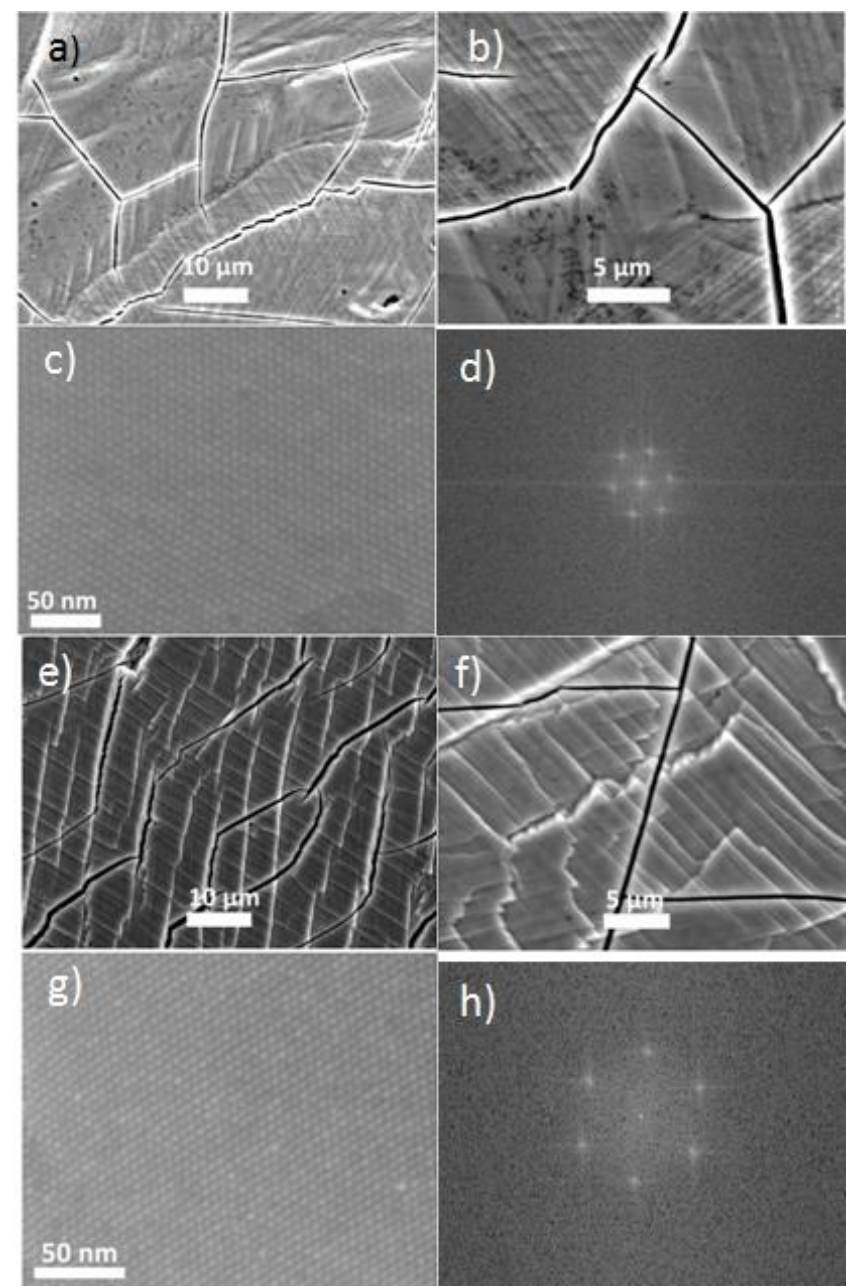

Figure 6. SEM images, a) and b), HRSEM image (c) of ${ }_{S D} \mathrm{Au}_{\mathrm{TMA}} \mathrm{NCs}$ film superlattice and fast-Fourrier-transformed pattern, d). SEM images, e), f) and HRSEM image, g) of POLYAu $\mathrm{Au}_{\mathrm{TMA}} \mathrm{NCs}$ and h) FFT image of the latter NCs.

In that case, the identification between SDAuTDT and POLYAuTDT NCs was possible thanks to the characteristic morphology of the supracrystals forming triangular shape and film supracrystals respectively. Herein no changes in the morphology of the supracrystals was observed (Figures $8 \mathrm{c}$ and $8 \mathrm{e}$ ), thus rendering more complicate to identify the nanocrystallinity segregation. In a previous study ${ }^{32,33}$ we demonstrated that a hierarchy in the supracrystal growth processes from amorphous-fcc films to shaped supracrystals existed. The fact that with hydrophilic SDAuTMA NCs we obtained film instead of shaped supracrystals - the case of hydrophobic SDAuTDT NCs permits to assume that the interactions between SDAuTMA NCs within the supracrystals are weaker than those existing in SDAuTDT NCs supracrystals. This could be due to the fact that the Autma NCs surface is positively charged. Furthermore, such process is inherent to mixed nanocrystal populations: in particular, as mentioned above, the interactions between NCs are stronger with SDAuTMA NCs than POLYAutma NCs. The facets of sDAutma NCs differ in shape, size, 
surface energy and atom density. Consequently, the crystallographic surface determins the electronic and bonding properties of the facets. The ability of POLYAuTMA NCs and SDAuTMA NCs to interact with their environment via the coating molecules is probably affected by the variation in the facet properties from one crystallographic surface to the other. The stronger interactions between SDAuTMA NCs, which facilitate phase segregation, are also favored by the fact that the facets present at them possess a relatively large surface area, compared to the ones at POLYAutMA NCs. ${ }^{36}$ In addition, it has been proposed that NPs can be regarded as 'superatoms' or 'supermolecules' in a colloidal nanoparticle solution and a size/shape selection and orientation adjustment procedure can be automatically executed when the solvent can provide dynamic conditions for NCs. It seemed that in the present case, water can also provide such conditions for selective self-assembly of the two different NP populations. ${ }^{42}$ These changes in the NCs interactions between SDAuTMA and POLYAuTMA NCs are expected to induce a change in the mechanical properties of such assemblies.
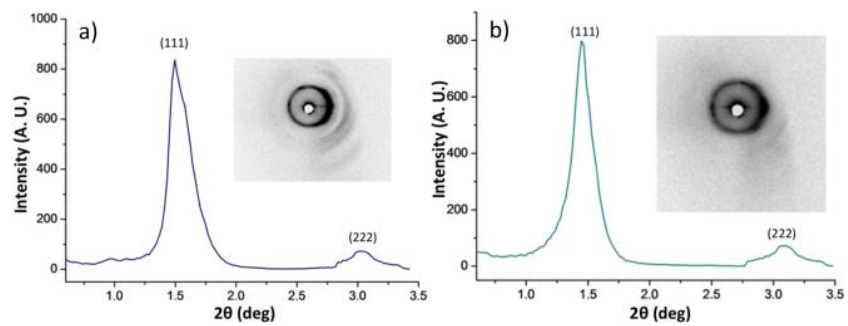

Figure 7. $1 \mathrm{D}$ and $2 \mathrm{D}$ (insets) $\mathrm{SAXRD}$ patterns of ${ }_{\mathrm{SD}} \mathrm{Au}_{\mathrm{TMA}}$ (a) and ${ }_{\mathrm{POLY}} \mathrm{Au}_{\mathrm{TMA}}$ (b) NCs self -assembled in fcc supracrystalline films.

\section{II.5. Mechanical properties of supracrystalline films.}

Several methods can be used to evaluate the mechanical properties of supracrystalline films prepared with NPs as constituting units. Here we deduced the Young's modulus value of the hydrophilic SDAuTMA and POLYAuTMA supracrystalline films using an atomic force microscope $(\mathrm{AFM})$. In a previous study, ${ }^{46}$ the validity of the nanoindentation measurements performed by using several AFM probes with different sizes and shapes against a nanoindentor $(\mathrm{N}$ indentor) was successfully tested. Here, mechanical measurements targeted to compare the values of Young's modulus of the films prepared using the hydrophilic POLYAutMa and SDAutMa NCs.

Figure 9 shows that the typical mechanical response of supracrystal films to the applied loads in the range of $100 \mathrm{nN}$ to $500 \mathrm{nN}$. Figures $9 \mathrm{a}$ and $9 \mathrm{~b}$ depict the AFM images of the residual marks remaining on POLYAuTMA and SDAuTMA supracrystal surfaces after indentations, respectively. It is found that when applying the same amount of load $(\sim 400 \mathrm{nN})$, the indentation traces are much deeper for POLYAuTMA supracrystal film than SDAuTMA supracrystal film (Figure 9c). Moreover, for the same applied load, the cantilever is displaced more on the surface of POLYAuTMA than SDAuTMA supracrystals as demonstrated in Figure 9d. All these results illustrate that qualitatively, supracrystals of POLYAuTMA NCs have a lower Young's modulus than supracrystals composed of SDAuTMA NCs. This result was also confirmed quantitatively, when the Young's modulus of each supracrystals was calculated by using the Oliver-Pharr model for almost 50 measurements. As a result of averaging 50 measurements (Figure 10), the Young's modulus of sDAutma and POLYAutma NCs were found as $315 \pm 82 \mathrm{MPa}$ and $172 \pm 39 \mathrm{MPa}$, respectively (Table 1).

Previously, it was found that the Young's modulus of various hydrophobic supracrystals depend on the nanocrystallinity, ${ }^{41}$ the supracrystal growth mechanism, ${ }^{48}$ the type of NPs, the interparticle distance, the material used ${ }^{41,49}$ and the type of the coating agent. ${ }^{47}$ Note that some controversy exists concerning the change in the Young's modulus with the NC size. ${ }^{47,49}$ In this study, the size of NCs $(5 \mathrm{~nm})$, and the type of the coating agent (TMA) are the same for both supracrystals of SD and POLY Au NCs, while the interparticle distances between NCs at the corresponding supracrystalline films remained quite similar (2.2-nm and 2.4-nm respectively). The major difference between the two samples studied was the nanocrystallinity of NCs. Consequently, the difference of Young's modulus between supracrystals of SDAuTMA and POLYAuTMA NCs is assigned to the difference in their nanocrystallinities (for the same average NCs diameters, $\delta$ values and coating agents). As shown above, the electron diffraction pattern of SDAuTMA NCs exhibits arc patterns (Figure 2h) and that indicates an apparent orientation ordering of nanocrystals. This orientational ordering, implying an atomic alignment between these NCs, is related to strong interactions between nanocrystals of this type. For POLYAuTMA NCs, the specific arc patterns disappeared (Figure $2 \mathrm{j}$ ), illustrating that these NCs have no orientational (but only translational) ordering.
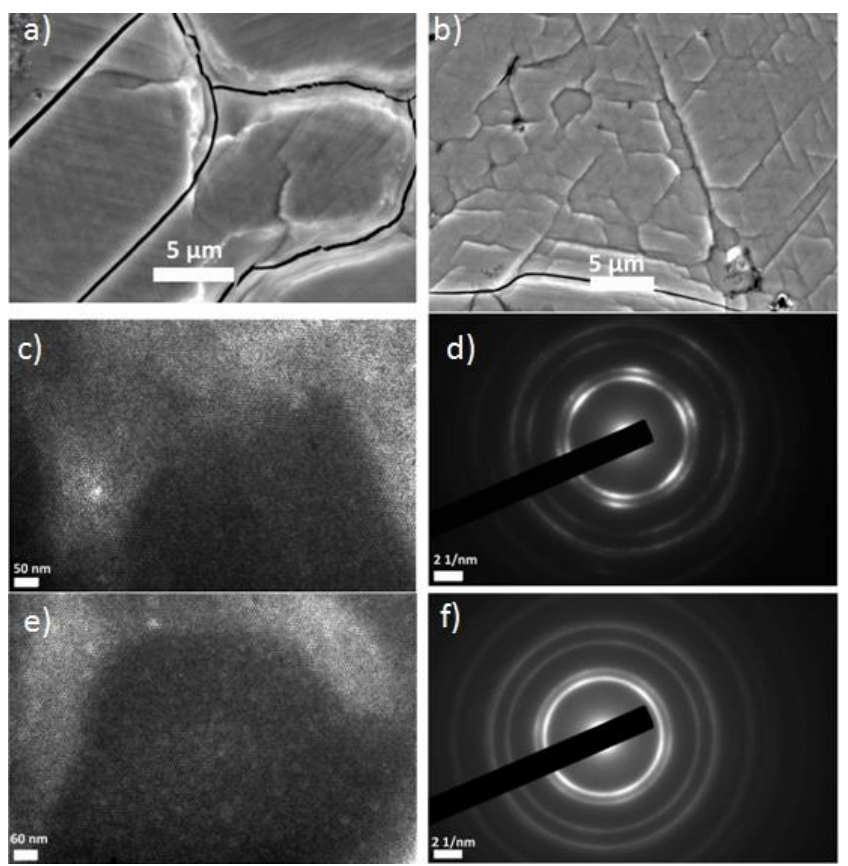

Figure 8. a) and b) SEM images of TMA-mix (50/50 ${ }_{\mathrm{POLY}} \mathrm{Au}_{\mathrm{TMA}}$ and ${ }_{\mathrm{SD}} \mathrm{Au}_{\mathrm{TMA}}$ NCs) film superlattices. c) and e) TEM images of two different 3- to 4-layer thick superlattices of TMA-mix Au NPs, with corresponding electrondiffraction patterns at d) and f) respectively. Scale bars at c) and e) are 50 and $60 \mathrm{~nm}$ respectively.

This random orientation was associated to weaker interactions between nanocrystals compared to the case of SD Au nanoparticles. As a result, the change in the Young's modulus by a factor of two between SDAuTMA and POLYAutMA NCs $(315 \pm 82$ and $172 \pm 39 \mathrm{MPa}$ respectively) is attributed to the degree of NCs ordering.

We previously observed ${ }^{47}$ a marked enhancement of the Young's modulus in supracrystals made up of 5-nm hydrophobic SDAuDDT NCs with respect to those formed using polycrystalline ones. The Young's moduli of supracrystals composed of SDAuDDT and POLYAuDDT NCs were measured as $550 \pm 160 \mathrm{MPa}$ and $25 \pm 9 \mathrm{MPa}$, respectively. That drastic change was attributed to different packing behaviors: while SD NCs exhibit both translational and orientational ordering in the superlattices, their POLY counterparts behave like spheres with no orientational ordering. If we compare the Young's moduli of supracrystals consisted of SD 5-nm Au NCs differing by their coating 
agents, i.e. TMA (hydrophilic) and DDT (hydrophobic), a twofold difference between their values is observed (315 MPa and $550 \mathrm{MPa}$, for quite similar interparticle distances, $2.2 \mathrm{~nm}$ and $2.4 \mathrm{~nm}$ respectively). This difference could be attributed to the fact the terminal group of TMA is positively charged inducing weaker interactions between NCs.

For POLY 5-nm Au NCs, the Young's Modulus markedly changed from $172 \mathrm{MPa}$ to $25 \mathrm{MPa}$ by replacing the coating agent from TMA to DDT while keeping the interparticle distances similar. Here, for both POLYAuTMA NCs and POLYAuDDT NCs, no orientational ordering appeared. However, the influence of the terminal group could still play a role. Consequently, the difference in Young's modulus could be due to a steric effect. More specifically, with POLYAuTMA NCs, such steric effect seems indeed to take place. This is because of the ammonium chloride moiety which might block the NCs in a certain configuration. Nevertheless, the methyl groups of the POLYAuDDT NCs could possibly rotate more easily under the strength of the AFM tip. This is a possible reason for the decrease in the Young's modulus by a factor of 2 between hydrophilic SDAuTMA and POLYAuTMA NCs (315 \pm 82 and $172 \pm 39 \mathrm{MPa}$, respectively), whereas the drop is higher than an order of magnitude between hydrophobic SDAuDDT and POLYAuDDT NCs $(550 \pm 160 \mathrm{MPa}$ to $25 \pm 9 \mathrm{MPa}$, respectively) for a similar interparticle distance among nanocrystals. Therefore, it seems reasonable to conclude that the Young's modulus of supracrystals depends on the crystalline structure of NCs used as building blocks, as well as on the steric effect of the coating agent and the surface charge of the NCs.
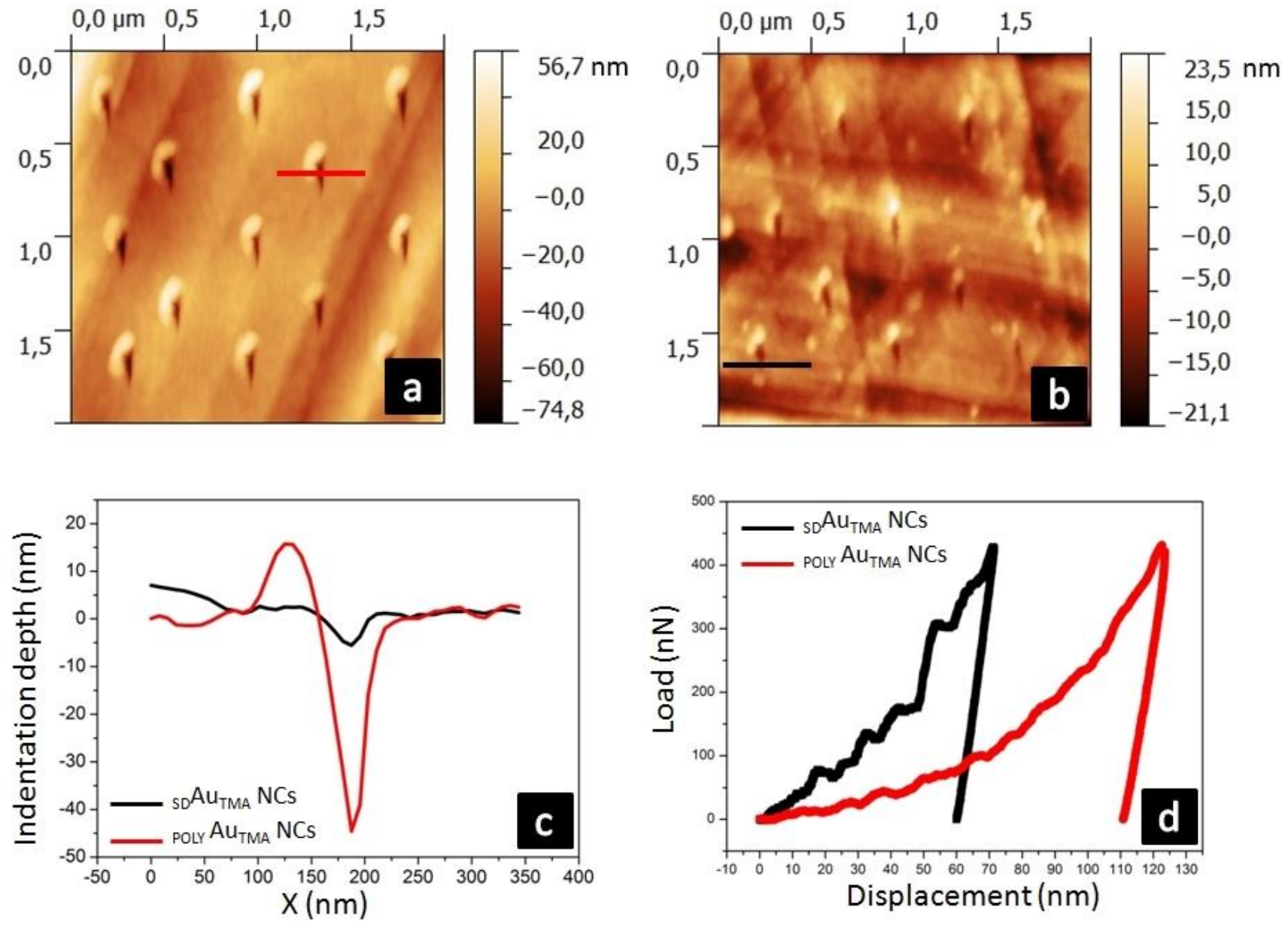

Figure 9. a) A set of indentation prints obtained by AFM imaging in acoustic mode after indenting on supracrystal film of PoLYAu ${ }_{\mathrm{TMA}}$ with different applied loads in the range of $100 \mathrm{nN}$ to $500 \mathrm{nN}$. b) A set of indentation prints obtained on supracrystal film of ${ }_{\mathrm{SD}} A \mathrm{u}_{\mathrm{TMA}}$ for the applied loads between $100 \mathrm{nN}$ and 500 $\mathrm{nN}$. c) The line profile of the indentation trace on the surface of ${ }_{\mathrm{POLY}} \mathrm{Au}_{\mathrm{TMA}}$ supracrystal (red line) and ${ }_{\mathrm{SD}} \mathrm{Au}_{\mathrm{TMA}}$ supracrystal (black line) for the applied load of $400 \mathrm{nN}$. d) Comparison of the load-displacement curves between both types of supracrystal surfaces for the same applied load ( $400 \mathrm{nN})$ on the cantilever. 

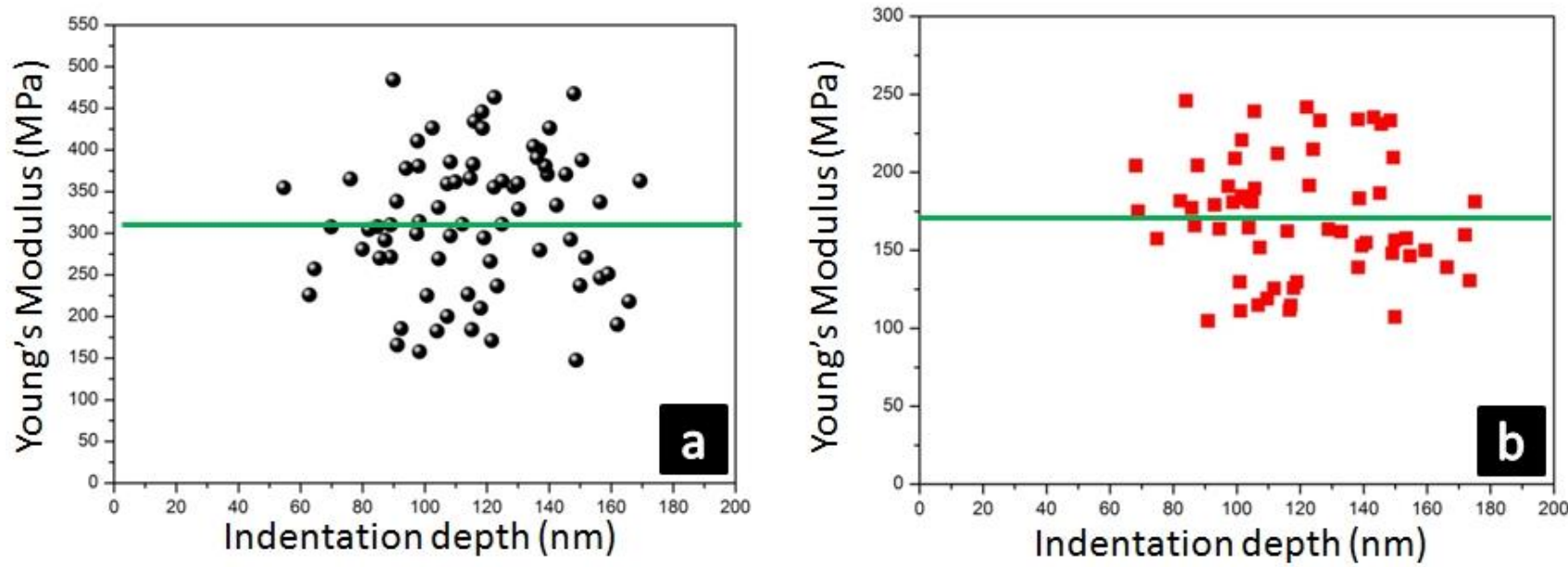

Figure 10. Plot of Young's modulus as a function of indentation depth measured for a) ${ }_{\mathrm{SD}} \mathrm{Au}_{\mathrm{TMA}}$ and b) ${ }_{\mathrm{POLY}} \mathrm{Au} \mathrm{TMA}_{\mathrm{TM}} \mathrm{NPs}$. The green lines show the average value of 50 measurements.

Table 1. Nanocrystal size (D), interparticle distance $\left(\delta_{\mathrm{pp}}\right)$, and Young's modulus of water soluble TMA-coated Au nanocrystals (sDA $\mathbf{u}_{\mathrm{TMA}}$ and ${ }_{\mathbf{P O L Y}} \mathbf{A \mathbf { u } _ { \mathrm { TMA } }} \mathrm{NCs}_{\text {) }}$.

Nanocrystal type

$\mathrm{D}(\mathrm{nm})$

$\delta_{\mathrm{pp}}(\mathrm{nm})$

Young's Modulus

(MPa)
SDAutma NCs

5.4

2.2

$315 \pm 82$

\section{polyAutma NCs}

5.2

2.4

$172 \pm 39$ 


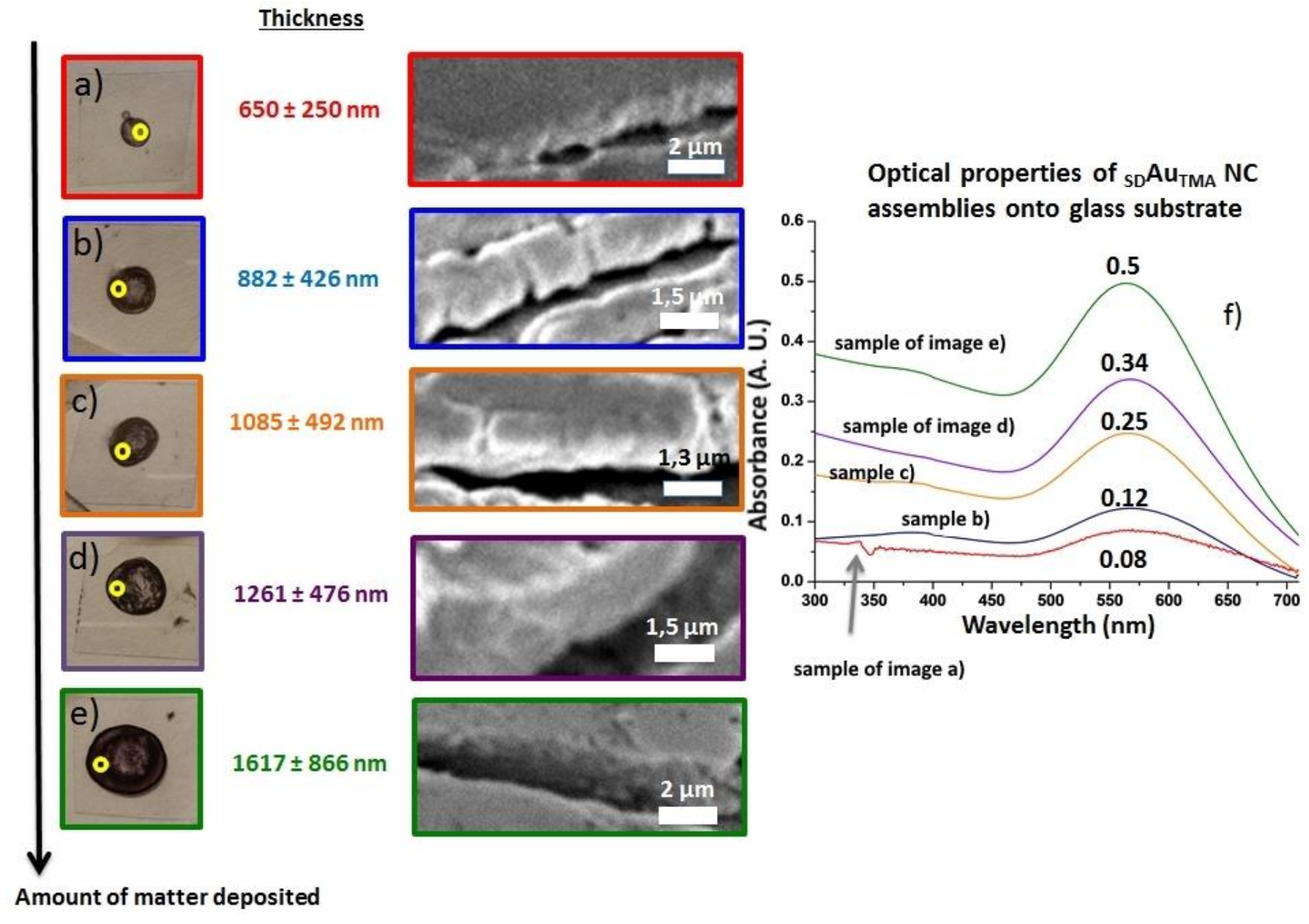

Figure 11. a), b) c), d) and e) Photographs of ${ }_{\mathrm{SD}} \mathrm{Au}_{\mathrm{TMA}}$ assemblies onto glass substrates of various thicknesses. f) UV-Vis spectra of those Au NP assemblies. Red curve-sample corresponds to sample of image a), blue curve - sample corresponds to sample of image b), orange curve-sample: image c), purple curvesample: image d), green curve-sample: image e). The yellow circle at images a)-e) denotes which area of the sample was used for the measurement of the thickness of the sample and of its optical properties. 
II.6. Optical properties of colloidal SDAuTMA and SDAuTMA NP assemblies deposited on a glass wafer

Considering the rather slight difference between the SDAuTMA and POLYAutMA NCs LSPR spectra (Figure 3) our study of the optical properties of thin films is carried out by using SDAuTMA NCs as building block of the fcc supracrystals. Various number of drops (each drop is $10 \mu \mathrm{l})$ of the colloidal solution ([SDAuTMA ]=10 mM) were drop-casted onto glass substrates. The samples were left 12 hours in air during the drying process. Different amounts were used, with the minimum quantity deposited being equal to $10 \mu \mathrm{l}$ of the aforementioned solution, while the sample with the largest quantity was of $90 \mu \mathrm{l}$ (that is, $10 \mu \mathrm{l}, 30 \mu \mathrm{l}, 50 \mu \mathrm{l}, 80 \mu \mathrm{l}$ and $90 \mu \mathrm{l}$ ). Figure 11 shows the patterns of the film produced by deposition of various amounts of the colloidal solution. The corresponding SEM images presented show formation of film having various thicknesses. An increase in the film thickness was clearly observed on increasing the amount of matter deposited. Obviously from the patterns, the SEM images and the measurements obtained by tilting the samples show that the films are inhomogeneous and a large dispersion of the thicknesses is obtained. Nevertheless we managed to perform consistent optical measurements. The LSPR spectra of such films were measured by using horizontally polarized light source while the incident light was kept parallel to the substrate. Figure 11 shows the corresponding LSPR spectra at various film thicknesses. The absorption spectra of the film differing by their thicknesses are centered at 568-nm and redshifted in comparison to the position of the colloidal Au NCs dispersed in aqueous solution (521-nm). These differences between the LSPR spectra at various thicknesses and the corresponding spectrum in colloidal solution are attributed to dipolar interactions between NCs. ${ }^{49}$ We have to mention that the position of the LSPR peak at Figure 11 does not change with the film thickness. At a first glance, these data seem to disagree with the trend noticed by producing nanoparticle films of $15 \mathrm{~nm} \mathrm{Au} \mathrm{NPs}$ encapsulated in silica. ${ }^{50}$ That inorganic spacer permitted to tune the distance between nanocrystals, and consequently the Au volume fraction by almost a factor of 3. Mulvaney and coworkers $^{50}$ observed that the LSPR peak was shifted to lower energy when the average distance between nanocrystals is rather low. On increasing this distance the shift was less pronounced and the LSPR peak position remained practically unchanged for shells equal or larger than $7 \mathrm{~nm}$. This corresponded to a $\mathrm{Au}$ volume fraction of 0.234 . In the present paper, the Au volume fraction is 0.248 (see eq. 16 at ref. 50, in our case the shell thickness corresponds to the interparticle distance, due to TMA coating). This result shows a rather good agreement between the data published in the work by Ung, Liz-Marzan and Mulvaney ${ }^{50}$ and those presented herein. Our results cleary demonstrate that the distance between nanocrystals is large enough to be able to not take into account the difference in dielectric constant between neighboring NPs and the glass substrate. Consequently the absorption spectra are almost insensitive to the total film thickness. A similar trend was also observed for rather thin $\mathrm{Ag} \mathrm{NCs} \mathrm{film}{ }^{51}$ and confirmed by simulation. ${ }^{52}$

For both solid film and colloidal solution, the LSPR intensity linearly increased with increasing the amount of matter as predicted by the Beer Lambert law (Figure S7). However, by comparison of the measured LSPR intensity obtained by using given amounts of matter either in colloidal solution or deposited onto the glass wafer, the slope of the intensity versus concentration function was larger in the case of the colloidal $\mathrm{Au}$ NPs. This was probably related to the heterogeneity in the film thickness and also to dipolar interaction between NCs. The unchanged absorption spectra with increasing the thickness of the film indicate that for relatively low thickness the surface of the film controlled its optical properties.

\section{Conclusions}

To summarize, dodecanethiol-coated Au NPs with either singlecrystalline or polycrystalline structure were synthesized. By a ligand exchange process that we elaborated, dodecanethiol molecules (DDT) were replaced by the positively charged N,N,Ntrimethyl(11-mercaptoundecyl) ammonium chloride, TMA. The SD and POLY 5-nm Au NCs coated with TMA (sDAuTMA and POLYAuTMA NCs respectively) were readily dispersed in water to form highly stable colloidal solutions. The Localized Surface Plasmon Resonance (LSPR) spectrum of POLYAuTMA NCs dispersed in aqueous solution was slightly broader than that obtained from SDAuTMA NCs. A slow water evaporation process produces films of either SDAuTMA or POLYAuTMA NCs or a mixture of both. These films are ordered in $f c c$ crystalline structures. The interparticle distance deduced from structural analysis remains rather similar by using either SDAuTMA or POLYAuTMA NCs $(2.2 \mathrm{~nm}$ and $2.4 \mathrm{~nm}$ respectively). Interestingly, the supracrystal growth of compositionally 'mixed' solutions provokes a nanocrystallinity segregation effect, i.e. SDAuTMA and POLYAuTMA NCs populations tend to segregate from each other to form ordered assemblies in distinct zones of the substrate. The mechanical stiffness of the supracrystals differing by their nanocrystallinity (sDAuTma and POLYAutma NCs) used as building block differed by a factor of 2 (315 and $172 \mathrm{MPa}$ respectively) whereas when using similar nanocrystals coated with hydrophobic surfactants (DDT) our previous data show that a difference by an order of magnitude was observed. These differences in the Young modulus were attributed to the terminal group of the coating agent characterized by both a positive charge and a steric constraint. Consequently this showed the influence of the surface chemistry on specific properties of supracrystals. The LSPR spectrum of SDAuTMA NCs deposited onto glass substrate was red shifted compared to that obtained in aqueous solution. This was assigned to dipolar interactions induced by the close vicinity of NCs. A linear dependence of the LSPR band intensity on the NCs concentration, as in the case of the optical properties of colloidal NP solutions, was obtained. This clearly confirmed, for small-sized supracrystals, no specific effects of the supracrystalline texture on the evolution of the optical properties with increasing the NCs amount measured.

\section{Experimental Section}

Chemicals. All chemical reagents were used without any further purification after their purchase. 1-dodecanethiol (>98\%), toluene (anhydrous, 99.8\%) and methanol (anhydrous, 99.8\%) were bought from Sigma-Aldrich. Borane tert-butylamine complex (97\%) and chlorotriphenylphosphine $\operatorname{gold}(\mathrm{I})$ (98\%) were purchased from Strem. N,N,N-trimethyl(11-mercaptoundecyl) ammonium chloride (TMA) was obtained from Prochima while ethyl acetate (RPE) was purchased from Carlos Erba Reagents. Ethanol (96\%) was obtained by VWR Chemicals. Millipore water $(18.2 \mathrm{M} \Omega)$ was used.

Au nanoparticle synthesis. The initial gold nanoparticle solution, containing both single-crystalline and polycrystalline particles, was prepared by employing a revisited protocol from Stucky and co-workers. ${ }^{37}$ Briefly, in a glovebox, $0.25 \mathrm{mmol}$ of $\left(\mathrm{Ph}_{3} \mathrm{P}\right) \mathrm{AuCl}$ were inserted in $25 \mathrm{ml}$ of toluene, together with $2.1 \mathrm{mmol}(500 \mu \mathrm{l})$ of dodecanethiol (solution A). In a separate vial, $5 \mathrm{mmol}$ of tert- 
butylamine borane complex were dissolved into $5 \mathrm{ml}$ of toluene (solution B). Both solutions were then placed at a preheated oil bath at $100^{\circ} \mathrm{C}$, in the glovebox, for $10 \mathrm{~min}$. Afterwards the solution $\mathrm{B}$ was added in the solution A and the new solution was heated for another $5 \mathrm{~min}$. A dark red color appeared fast upon the addition of solution $\mathrm{B}$, indicating the rapid reduction of the gold precursor and the onset of the nucleation stage. The product was allowed to cool down before its drying by using a mild flow of nitrogen gas for a few hours. $10 \mathrm{ml}$ of ethanol were then introduced and the resulting solution was centrifuged (2000 rpm, $3 \mathrm{~min}$ ). The supernatant was discarded and $25 \mathrm{ml}$ of toluene were added, together with $1 \mathrm{ml}$ of ethanol.

Nanocrystallinity segregation. The procedure to separate the single-crystalline Au NPs from the polycrystalline ones has already been reported in some of our previous works: ${ }^{36}$ The aforementioned solution was left for one week in a closed bottle (to avoid solvent evaporation). A black precipitate was isolated by centrifugation at $5000 \mathrm{rpm}$ for $10 \mathrm{~min}$ and it was dispersed into either hexane or toluene (single-crystalline NPs). The supernatant contained the polycrystalline NPs.

Samples for TEM were prepared by the deposition of a drop of the colloidal dispersions onto carbon-coated $\mathrm{Cu}$ grids. Conventional TEM images were acquired with a JEOL JEM 1011 microscope operating at an acceleration voltage of $100 \mathrm{kV}$. Electron diffraction patterns were obtained with the aforementioned microscope. The camera length was set to either 10 or $20 \mathrm{~cm}$. HRTEM images were obtained with a JEOL JEM 2011 apparatus operating at $200 \mathrm{kV}$. Scanning electron microscopy (SEM) and HRSEM imaging was carried out with JEOL JSM-5510LV and SEM-FEG Hitachi SU-70 microscopes respectively. Small-angle X-ray diffraction (SAXRD) measurements were carried out using a homemade system mounted on a rotating copper anode generator. Diffraction patterns were recorded on photostimulable imaging plates. Optical characterization was performed with a UV/vis/near-IR Cary 5000 spectrophotometer.

Ligand exchange. $5 \mathrm{~nm} \mathrm{Au}$ nanocrystals capped by dodecanethiol were treated with the positively charged TMA so as to become water soluble. For simplicity, nanoparticles differing by their crystalline phase and coated by TMA are abbreviated as SDAuTMa and POLYAutma (single-crystalline versus polycrystalline ones).

SDAuTMA NPs were produced by the following procedure: $8 \mathrm{mg}$ TMA were diluted in $200 \mu \mathrm{L}$ methanol. The TMA solution was then added in a solution of dodecanethiol-stabilized Au NPs in toluene $(1.5 \mathrm{~mL}, 1 \mathrm{mg} / \mathrm{mL})$. The new mixture was placed in an oil bath at $80{ }^{\circ} \mathrm{C}$ for $3 \mathrm{~h}$ under magnetic stirring. After cooling, the product was centrifuged ( $5000 \mathrm{rpm}, 5 \mathrm{~min})$, and the precipitate was washed with ethyl acetate $(3 \times 2 \mathrm{~mL})$. For further purification, the

(1) Murray, C. B.; Kagan, C. R.; Bawendi, M. G. Self-organization of CdSe Nanocrystallites into three-dimensional quantum dot superlattices. Science, 1995, 270, 1335-1338.

(2) Motte, L.; Billoudet, F.; Pileni, M. P. Self-Assembled monolayer of nanosized particles differing by their sizes. J. Phys. Chem., 1995, 99, 16425-16429.

(3) Pileni, M. P. Nanocrystal self-assemblies: Fabrication and collective properties. J. Phys. Chem. B 2001, 105, 3358-3371.

(4) Tracy, J. B.; Bawendi, M. G. Defects in CoO in oxidized cobalt nanoparticles dominate exchange biasing and exhibit anomalous magnetic properties. Phys. Rev. B 2006, 74, 184434. precipitate was diluted in $0.5 \mathrm{~mL}$ methanol and precipitated again by inserting $2 \mathrm{~mL}$ ethyl acetate. That procedure was repeated twice. In the end, the solid product was dried with a mild flow of $\mathrm{N}_{2}$ and diluted in $0.5 \mathrm{ml}$ of deionized water, to provide a dark red stable colloidal solution.

The preparation of POLYAuTMA NPs followed the same line: 11 mg TMA were dispersed in $200 \mu \mathrm{L}$ methanol. The TMA solution was then inserted into a solution of DDT-capped Au NPs in toluene $(1.5 \mathrm{ml}, 1.5 \mathrm{mg} / \mathrm{mL})$. Heating, stirring, washing and storage conditions were identical to the ones regarding the SDAuTMA NPs.

Modification of the silicon wafer. The procedure to turn the $\mathrm{Si}$ wafers hydrophilic involved their immersion in a 3:1 (v/v) mixture of $\mathrm{H}_{2} \mathrm{SO}_{4}(96 \%)$ and $\mathrm{H}_{2} \mathrm{O}_{2}(30 \%)$ for $30 \mathrm{~min}$; they were later rinsed with deionized water for several times.

AFM measurements. The Young's modulus of Au supracrystals made of monocrystalline and polycrystalline water soluble TMAcoated nanoparticles were measured with Microscopy (AFM) nanoindentation at room temperature by recording the displacement of the cantilever during its up and down movements on the surfaces while applying some loads to the AFM tip. Approximately 50 load-displacement curves were recorded at different locations of each supracrystal films for various applied loads ranging from $100 \mathrm{nN}$ to $500 \mathrm{nN}$. Finally, the Young's moduli of $\mathrm{Au}$ supracrystal films were calculated from the loaddisplacement curves by using the Oliver-Pharr $\operatorname{model}^{53,54}$ as described in detail in the Supporting Information.

\section{Corresponding author: mppileni@ orange.fr}

Notes: The authors declare no competing financial interest

\section{Acknowledgements}

The authors are grateful to S. Casale for HRTEM observations. P.A. Albouy is acknowledged for the SAXRD measurements. We thank D. Montero for HRSEM imaging. S. M. thanks all the members of the SUPRANANO group for their help and for fruitful discussions. This research project was funded from the Advanced Grant of the European Research Council under Grant Agreement 267129 .

Supporting Information. Additional HRTEM and HRSEM images, measurements of optical properties and analytical description of the AFM measurements.

\section{References}

(5) Pileni, M. P. Supracrystals of inorganic nanocrystals: An open challenge for new physical properties. Acc. Chem. Res. 2008, 41, 1799-1809.

(6) Pileni, M. P. Supra- and Nanocrystallinity: Specific properties related to crystal growth mechanisms and nanocrystallinity. Acc. Chem. Res. 2012, 45, 1965-1972.

(7) Stoeva, S. I.; Prasad, B. L. V.; Uma, S.; Stoimenov, P. K.; Zaikovski, V.; Sorensen. C. M.; Klabunde, K. J. Face-centered cubic and hexagonal closed-pack nanocrystal superlattices of gold nanoparticles prepared by different methods. J. Phys. Chem. B, 2003, 107, 7441-7448.

(8) Compton, O. C.; Osterloh, F. E. Evolution of size and shape in the colloidal crystallization of gold nanoparticles. J. Am. Chem. Soc. 2007, 129, 7793-7798. 
(9) Goubet, N.; Portalès, H.; Yan, C.; Arfaoui, I.; Albouy P. A.; Mermet. A.; Pileni, M. P. Simultaneous growths of gold colloidal crystals. J. Am. Chem. Soc., 2012, 134, 3714-3719.

(10) Whetten, R. L.; Shafigullin, M. N.; Khoury, J. T.; Schaaft, T. G.; Vezmar, I.; Alvarez. M. M.; Wilkinson A.; Crystal structures of molecular gold nanocrystal arrays. Acc. Chem. Res. 1999, 32, 397-406.

(11) Yang, Z.; Wei, J.; Bonville, P.; Pileni, M. P. Beyond entropy: Magnetic forces induce formation of quasicrystalline structure in binary nanocrystal superlattices. J. Am. Chem. Soc., 2015, 137, 4487-4493.

(12) Gauvin, M.; Yang, N.; Yang, Z.; Arfaoui, I.; Pileni, M. P. Hierarchical mechanical behaviour of cobalt supracrystals related to nanocrystallinity. Nano Res. 2015, 8, 3480-3487.

(13) Goubet, N.; Yang, J.; Albouy, P. A.; Pileni, M. P. Spontaneous formation of high-index planes in gold single domain nanocrystal superlattices. Nano. Lett., 2014, 14, 6632-6638.

(14) Talapin, D. V.; Shevchenko, E. V.; Murray, C. B.; Titov, A. V.; Kral, P., Dipole-dipole interactions in nanoparticle superlattices. Nano Lett. 2007, 7, 1213-1219.

(15) Tao, A. R.; Ceperley, D. P.; Sinsermsuksakul, P.; Neureuther, A. R.; Yang, P. Self-organized silver nanoparticles for threedimensional plasmonic crystals. Nano Lett. 2008, 8, 4033-4038. (16) Maier, S. A.; Kik, P. G.; Atwater, H. A.; Meltzer, S.; Harel, E.; Koel. B. E.; Requicha, A. G.; Local detection of electromagnetic energy transport below the diffraction limit in metal nanoparticle plasmon waveguides. Nat. Mater. 2003, 2, 229232.

(17) Zayats, M.; Kharitonov, A. B.; Pogorelova, S. P.; Lioubashevksi, O.; Katz. E.; Wilner, I. Probing photoelectrochemical processes in Au-CdS nanoparticle arrays by surface plasmon resonance: Application for the detection of acetylcholine esterase inhibitors. J. Am. Chem. Soc. 2003, 125, 16006-16014.

(18) Zie, N.; Petukhova, A.; Kumacheva, E. Properties and emerging applications of self-assembled structures made from inorganic nanoparticles. Nat. Nanotechnol. 2010, 5, 15-25.

(19) Boles, M. A.; Engel, M.; Talapin, D. V. Self-assembly of colloidal nanocrystals: From intricate structures to functional materials. Chem. Rev. 2016, 116, 11220-11289.

(20) Kowalczyk, B.; Walker, D. A.; Soh, S.; Grzybowski, B. A.; Nanoparticle supracrystals and layered supracrystals as chemical amplifiers. Angew. Chem. Int. Ed. 2010, 49, 5737-5741.

(21) Wang, S. H.; Yao, H.; Sato, S.; Kimura, K. Inclusion-watercluster in a three-dimensional superlattice of gold nanoparticles. $J$. Am. Chem. Soc. 2004, 126, 7438-7439.

(22) Kalsin, A. M.; Fialkowski, M.; Paszewski, M.; Smoukov, S. K.; Bishop, K. J. M.; Grzybowski, B. A. Electrostatic selfassembly of binary nanoparticle crystals with a diamond-like lattice. Science 2006, 312, 420-424.

(23) Macfarlane, R. J.; Lee, B.; Jones, M. R.; Harris, N.; Schatz, G. C.; Mirkin, C. A., Nanoparticle superlattice engineering with DNA. Science, 2011, 334, 204-208.

(24) Kumar, A.; Zhang, X.; Liang, X.-J. Gold nanoparticles: emerging paradigm for targeted drug delivery system. Biotechnol. Adv. 2013, 31, 593-606

(25) Mieszawska, A. J.; Mulder, W. J. M.; Fayad, Z. A.; Cormode, D. P. Multifunctional gold nanoparticles for diagnosis and therapy of disease. Mol. Pharmaceutics 2013, 10, 831-847

(26) Lim, Z. Z. J.; Li, J. E. J.; Ng, C. T.; Yung, L. Y. L.; Bay, B. H. Acta Pharmacol. Sin. 2011, 32, 983-990.

(27) Wang, L.; Albouy, P.-A.; Pileni, M. P. Synthesis and selfassembly of charged Au nanocrystals in aqueous solution. Chem. Mater. 2015, 27, 4431-4440.

(28) Jana, N. R.; Peng, X. G. Single-phase and gram-scale routes toward nearly monodisperse $\mathrm{Au}$ and other noble metal nanocrystals. J. Am. Chem. Soc. 2003, 125, 14280-14281.

(29) Polavarapu, L.; Mourdikoudis, S.; Pastoriza-Santos, I.; PerezJuste, J. Nanocrystal engineering of noble metals and metal chalcogenides: controlling the morphology, composition and crystallinity. CrystEngComm 2015, 17, 3727-3762.

(30) van Embden J.; Chesman, A. S. R.; Jasienak, J. J. The heatup synthesis of colloidal nanocrystals. Chem. Mater. 2015, 27, 2246-2285.

(31) Koczkur, K. M.; Mourdikoudis, S.; Polavarapu, L.; Skrabalak, S. E. Polyvinylpyrrolidone (PVP) in nanoparticle synthesis. Dalton Trans. 2015, 44, 17883-17905.

(32) Wan, Y. F.; Goubet, N.; Albouy, P. A.; Pileni, M. P. Hierarchy in Au nanocrystal ordering in supracrystals: a potential approach to detect new physical properties. Langmuir, 2013, 29, $7456-7463$.

(33) Liu, T.; Jiang, P.; You, Q.; Ye, S. Five-fold twinned pentagonal gold nanocrystal structure exclusively bounded by $\{110\}$ facets. CrystEng Comm, 2013, 15, 2350-2353

(34) Zhang, Q.; Xie, J.; Yu, Y.; Yang, J.; Lee, J. Y. Tuning the crystallinity of Au nanoparticles. Small, 2010, 6, 523-527.

(35) Goubet, N.; Tempra, I.; Yang, J.; Soavi, G.; Polli, D.; Cerullo, G.; Pileni, M. P. Size and nanocrystallinity controlled gold nanocrystals : synthesis, electronic and mechanical properties. Nanoscale, 2015, 7, 3237-3246.

(36) Portales, H.; Goubet, N.; Sirotkin, S.; Duval, E.; Mermet, A.; Albouy, P.-A.; Pileni, M. P. Crystallinity segregation upon selective self-assembling of gold colloidal single nanocrystals. Nano Lett. 2012, 12, 5292-5298.

(37) Zheng, N.; Fan, J.; Stucky, G. D. ; One-step one-phase synthesis of monodisperse noble-metallic nanoparticles and their colloidal crystals. J. Am. Chem. Soc. 2006, 128, 6550-6551.

(38) de la Presa, P.; Rueda, T.; Morales, M. P.; Hernando, A. Ligand exchange in gold-coated FePt nanoparticles. IEEE Trans. Magn. 2008, 44, 2816-2819.

(39) Yu, K. ; Ng, P. ; Ouyang, J. ; Zaman, M. B. ; Abulrob, A. ; Baral, T. N. ; Fatehi, D. ; Zakubek, Z. J. ; Kingston, D. ; Wu, X. ; Liu, X. ; Hebert, C. ; Leek, D. M. ; Whitfield, D. M. ; Lowtemperature approach to highly emissive copper indium sulfide colloidal nanocrystals and their bioimaging applications. ACS Appl. Mater. Interf. 2013, 5, 2870-2880.

(40) Wan, Y.; Portales, H.; Goubet, N.; Mermet, A.; Pileni, M. P. Impact of nanocrystallinity segregation on the growth and morphology of nanocrystal superlattices. Nano Res. 2013, 6, 611618.

(41) Goubet, N. ; Yan, C. ; Polli, D. ; Portales, H. ; Arfaoui, I. ; Cerullo, G. ; Pileni, M. P. Modulating physical properties of isolated and self-assembled nanocrystals through change in nanocrystallinity. Nano Lett. 2013, 13, 504-508.

(42) Brown, L. O.; Hutchison, J. E.; Formation and electron diffraction studies of ordered 2-D and 3-D superlattices of aminestabilized gold nanocrystals. J. Phys. Chem. B 2001, 105, 89118916.

(43) Quan, Z.; Fang, J. Superlattices with non-spherical building blocks. Nano Today 2010, 5, 390-411.

(44) Harfenist, S. A.; Wang, Z. L.; Alvarez, M. M.; Vezmar, I.; Whetten, R. L. Highly oriented molecular Ag nanocrystal arrays. J. Phys. Chem. 1996, 100, 13904-13910.

(45) Xie, S. ; Zhou. X. ; Han, X. ; Kuang, Q. ; Jin, M. ; Jiang, Y. ; Xie, Z. ; Zheng, L. Supercrystals from crystallization of octahedral MnO nanocrystals. J. Phys. Chem. C 2009, 113, 19107-19111. (46) Gauvin, M. ; Yang, N. ; Barthel, E. ; Arfaoui, I. ; Yang, J. ; Albouy, P.-A. ; Pileni, M. P. Morphology, nanocrystallinity and elastic properties of single domain $\varepsilon$-Co supracrystals. J. Chem. C 2015, 119, 7483-7490.

(47) Gauvin, M.; Wan. Y.; Arfaoui, I.; Pileni, M. P., Mechanical properties of $\mathrm{Au}$ supracrystals tuned by flexible ligand interactions. J. Phys. Chem. C., 2014, 118, 5005-5012.

(48) Yan, C.; Arfaoui, I.; Goubet, N.; Pileni, M. P. Soft supracrystals of $\mathrm{Au}$ nanocrystals with tunable mechanical properties. Adv. Funct. Mater. 2013, 23, 2315-2321.

(49) Wei, J.; Schaeffer, N.; Albouy, P. A.; Pileni, M. P. Surface plasmon resonance properties of silver nanocrystals differing in 
size and coating agent ordered in 3D supracrystals. Chem. Mater. 2015, 27, 5614-5621.

(50) Ung, T.; Liz-Marzan, L. M.; Mulvaney, P. Optical properties of thin films of $\mathrm{Au} @ \mathrm{SiO}_{2}$ particles. J. Phys. Chem. B. 2001, 105, 3441-3452.

(51) Wei, J.; Schaeffer, N.; Pileni, M. P., Ag nanocrystals: 1. Effect of ligands on plasmonic properties. J. Phys. Chem. B, 2014, 118, 14070-14075.

(52) Wei, J.; Yang, P.; Portales, H.; Pileni, M. P., Collective surface plasmon resonances in two-dimensional assemblies of $\mathrm{Au}$ and $\mathrm{Ag}$ nanocrystals: Experiments and discrete dipole approximation simulation. J. Phys. Chem. C, 2016, 120, $13732-13738$

(53) Oliver, W. C.; Pharr, G. M. An improved technique for determining hardness and elastic modulus using load and displacement sensing indentation experiments. J. Mater. Res. 1992, 7, 1564-1583.

(54) Oliver, W. C.; Pharr, G. M. Measurement of hardness and elastic modulus by instrumented indentation: advances in understanding and refinements to methodology. J. Mater. Res. 2004, 19, 3-20.

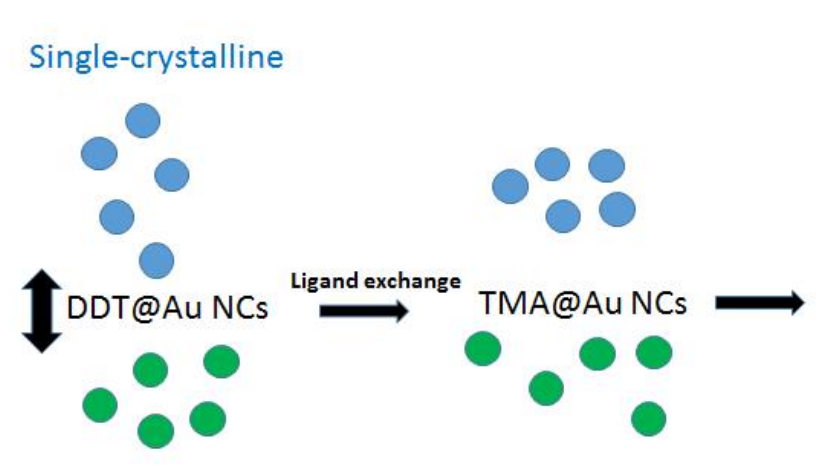

Polycrystalline

Hydrophobic

Hydrophilic

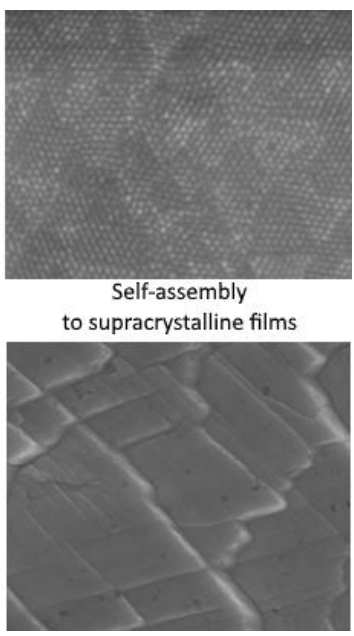

ToC Figure: 5-nm Au nanocrystals with well-defined nanocrystallinity, being transferred from organic solvent to aqueous medium and self-assembled to supracrystals 